\title{
Correction to: High Performance Computing
}

\author{
Michèle Weiland (D), Guido Juckeland (D), Sadaf Alam (D), \\ and Heike Jagode (D)
}

\section{Correction to:}

\section{Weiland et al. (Eds.) in: High Performance Computing,} LNCS 11887, https://doi.org/10.1007/978-3-030-34356-9

In the original version of this LNCS volume, four papers were erroneously released as open access papers. This has been corrected to only two papers - papers 5 and 7 .

The updated version of these chapters can be found at https://doi.org/10.1007/978-3-030-34356-9_5 https://doi.org/10.1007/978-3-030-34356-9_7 https://doi.org/10.1007/978-3-030-34356-9_23

https://doi.org/10.1007/978-3-030-34356-9_41

https://doi.org/10.1007/978-3-030-34356-9 